\title{
SERIES THAT CONVERGE ON SETS OF NULL DENSITY
}

\author{
R. ESTRADA AND R. P. KANWAL
}

\begin{abstract}
It is shown that a series of positive terms that converges on all sets of null density should be convergent. Using this result we construct examples of complete topological vector spaces that are proper subspaces of a Banach space, but whose dual spaces coincide with the dual space of the Banach space.
\end{abstract}

1. Introduction. We establish that a series of positive terms that converges on each set of null density has to be convergent.

In $\$ 2$ we introduce the relevant definitions and notation and give a proof of the result. In the third section, as an application, we construct complete topological vector spaces $X(p, q)$ for $1 \leqslant p<q<\infty$ which are proper subspaces of the Lebesgue space $l^{q}$, but whose dual spaces coincide with the space $l^{q^{\prime}}\left(1 / q+1 / q^{\prime}=\right.$ $1)$, dual of $l^{q}$.

2. The basic result. Let $E$ be a subset of the set of natural numbers $\mathbf{N}=$ $\{1,2,3, \ldots\}$. For each $n \in \mathbf{N}$ the $n$th order density of $E$ is defined as

$$
d_{n}(E)=n^{-1}|A(n) \cap E|,
$$

where the bars indicate the number of elements of a set and where

$$
A(n)=\{1, \ldots, n\} \text {. }
$$

The density of $E$ is defined as

$$
d(E)=\lim _{n \rightarrow \infty} d_{n}(E),
$$

whenever the limit exists. Not every set $E \subseteq \mathbf{N}$ has a density, although the upper and lower densities, $\bar{d}(E)$ and $\underline{d}(E)$, obtained by replacing the limit by the upper and lower limits, respectively, always exist.

The set function $d$ is finitely additive, namely,

$$
d(E \cup F)=d(E)+d(F),
$$

if $E$ and $F$ are disjoint sets that possess a density. Observe that the sets of null density form a ring of sets: If both $E$ and $F$ have null density then so does $E \cup F$.

EXAMPLE. The series $\sum_{n=1}^{\infty} 1 / n$ diverges. It is easy to show that for this series there are subsets $E$ of $\mathrm{N}$ of null density for which the series $\sum_{n \in E} 1 / n$ also diverges. We

Received by the editors July 3, 1985.

1980 Mathematics Subject Classification. Primary 40A05, 46A15, 46A45. 
can take, for instance, $E$ as the set of prime numbers or as the set $\{[[n \log n]]$ : $n \in \mathbf{N}\}$, where $[[x]]$ denotes the integral part of $x \in \mathbf{R}$. Theorem 1 says that the same is true for any divergent series.

For future reference we introduce the sets

$$
\begin{gathered}
B(n)=\{n, n+1, n+2, \ldots\}, \\
J(n ; j)=\{j, j+n, j+2 n, \ldots\}, \quad 1 \leqslant j \leqslant n .
\end{gathered}
$$

Observe that

$$
d(J(n ; j))=1 / n
$$

In fact, if $m \geqslant k \geqslant n$, then

$$
d_{m}(J(n ; j) \cap B(k)) \leqslant 1 / n,
$$

since

$$
d_{m}(J(n ; j) \cap B(k)) \leqslant \sup \left\{\frac{1}{k}, \frac{2}{k+n}, \frac{3}{k+2 n}, \ldots\right\}
$$

THEOREM 1. Let $\left(a_{n}\right)$ be a sequence of nonnegative real numbers. If for every set of null density $E$ the series $\sum_{n \in E} a_{n}$ converges, then the series $\sum_{n=1}^{\infty} a_{n}$ is also convergent.

Proof. We shall show that if the complete series is divergent then we can construct a set $E$ of null density on which the series diverges as well.

To simplify the analysis we put

$$
\mu(E)=\sum_{n \in E} a_{n}, \quad E \subseteq \mathbf{N} .
$$

If the complete series diverges, then $\mu(\mathbf{N})=\infty$. Hence we can choose $m_{1}$ such that

$$
\mu\left(A\left(m_{1}\right)\right) \geqslant 1 \text {. }
$$

Set $E(1)=A\left(m_{1}\right)$.

Choose $n_{1} \geqslant 4$ such that

$$
d_{n_{1}}(E(1)) \leqslant 1 / 4 \text {. }
$$

Since the series $\sum_{n=1}^{\infty} a_{n}$ diverges, then for $k$ fixed at least one of the series $\mu(J(k ; j))=\sum_{n \in J(k ; j)} a_{n}$ diverges. Choose $j$ such that $\mu(J(4 ; j))=\infty$ and let $m_{2}>n_{1}$ such that

$$
\mu\left(J(4 ; j) \cap B\left(n_{1}\right) \cap A\left(m_{2}\right)\right) \geqslant 1 / 2 .
$$

We put then

$$
E(2)=E(1) \cup\left[J(4 ; j) \cap B\left(n_{1}\right) \cap A\left(m_{2}\right)\right] .
$$

Observe that if $n \geqslant n_{1}$ then

$$
\begin{aligned}
d_{n}(E(2)) & \leqslant d_{n_{1}}(E(1))+d_{n}\left(J(4 ; j) \cap B\left(n_{1}\right)\right) \\
& \leqslant 1 / 4+1 / 4=1 / 2 .
\end{aligned}
$$

We can continue with this process to obtain sequences $\left(n_{k}\right)$ and $\left(m_{k}\right)$ such that

$$
m_{1}<n_{1}<m_{2}<n_{2}<\cdots<m_{k}<n_{k}<m_{k+1}<\cdots \text {, }
$$


as well as sets $E(1) \subseteq E(2) \subseteq E(3) \subseteq \cdots$ such that

$$
\begin{gathered}
E(k) \subseteq A\left(m_{1}\right) \cup\left[B\left(n_{1}\right) \cap A\left(m_{2}\right)\right] \cup \cdots \cup\left[B\left(n_{k-1}\right) \cap A\left(m_{k}\right)\right], \\
\mu\left(E(k) \cap\left[B\left(n_{k-1}\right) \cap A\left(m_{k}\right)\right]\right) \geqslant 1 / k, \\
d_{n}(E(k)) \leqslant 1 / k, \quad n \geqslant n_{k-1} .
\end{gathered}
$$

In fact, the construction can be made by using an inductive procedure. If $n_{1}, \ldots, n_{k-1}, m_{1}, \ldots, m_{k}, E(1), \ldots, E(k)$ have already been constructed, we select $n_{k}>2(k+1)$ such that

$$
d_{n_{k}}(E(k)) \leqslant 1 / 2(k+1) .
$$

Let $j$ be such that $\mu(J(2(k+1) ; j))=\infty$ and let $m_{k+1}$ be such that

$$
\mu\left(J(2(k+1) ; j) \cap B\left(n_{k}\right) \cap A\left(m_{k+1}\right)\right) \geqslant 1 /(k+1) .
$$

We then define

$$
E(k+1)=E(k) \cup\left[J(2(k+1) ; j) \cap B\left(n_{k}\right) \cap A\left(m_{k+1}\right)\right] .
$$

It is clear that (2.14), (2.15) and (2.16) are satisfied for $k+1$, while for (2.17) we observe that, if $n \geqslant n_{k}$,

$$
\begin{aligned}
d_{n}(E(k+1)) & \leqslant d_{n_{k}}(E(k))+d_{n}\left(J(2(k+1) ; j) \cap B\left(n_{k}\right)\right) \\
& \leqslant \frac{1}{2(k+1)}+\frac{1}{2(k+1)} \leqslant \frac{1}{k+1} .
\end{aligned}
$$

Let

$$
E=\bigcup_{k=1}^{\infty} E(k)
$$

Then, for each $k$,

$$
\mu(E) \geqslant \mu(E(k)) \geqslant 1+1 / 2+\cdots+1 / k,
$$

and hence

$$
\mu(E)=\sum_{n \in E} a_{n}=\infty .
$$

On the other hand, from the construction of the sets $E(k)$ we shall have

$$
d_{n}(E) \leqslant \frac{1}{k+1}, \quad n \geqslant n_{k} .
$$

This shows that $d(E)=0$, as desired.

3. An application. In this section we present an application of Theorem 1 to the construction of certain topological vector spaces $X(p, q)$ that are proper subspaces of the Lebesgue space $l^{q}$ but whose dual space coincides with the dual of the Lebesgue space. Our notation is the standard one; see $[1,2]$ for instance.

Let $X$ and $Y$ be complete topological vector spaces. Suppose that $X \subseteq Y$, the inclusion is continuous and $X$ is dense in $Y$. By duality, the dual space $Y^{*}$ of $Y$ can be considered as a subspace of the dual space $X^{*}$ of $X$. In case $X^{*}=Y^{*}$ we shall say that the pair $(X, Y)$ is an identification; if, furthermore, $X$ is a proper subspace of $Y$, we shall say that the identification is proper. 
It is easy to show the existence of proper identifications. In fact, let $X$ be a nonreflexive Banach space and let $Y_{0}$ be the vector space $X$ with the weak topology induced by $X^{*}$. Since $X$ is nonreflexive, the space $Y_{0}$ cannot be complete. If $Y$ is a completion of $Y_{0}$ then the pair $(X, Y)$ is a proper identification.

As the following result shows, however, certain kinds of proper identifications are not possible.

LEMMA. There are no proper identifications $(X, Y)$ of two Banach spaces.

Proof. Let $\left(X,\|\|_{1}\right)$ and $\left(Y,\|\|_{2}\right)$ be two Banach spaces. Let us suppose that $X \subseteq Y$, the inclusion is continuous, $X$ is dense in $Y$ and $X^{*}=Y^{*}$. We are going to show that $X=Y$, so that the identification is not proper.

Indeed, both dual spaces $X^{*}$ and $Y^{*}$ are Banach spaces. If $i: Y^{*} \rightarrow X^{*}$ is the inclusion, it follows that $i$ is (1-1) and continuous, but since it is onto, use of the open mapping theorem gives that $i$ is bicontinuous. Therefore the norms of $X^{*}$ and $Y^{*}$ are equivalent and this implies that the same is true of the norms \|\|$_{1}$ of $X$ and \|\|$_{2}$ of $Y$. But then $X$ is dense in $\left(Y,\|\|_{2}\right)$ and complete with the norm \|\|$_{2}$, and hence $X=Y$.

As is clear, we can weaken the assumptions in the Lemma. It is enough to suppose, for instance, that both $X$ and $Y$ are strict inductive limits of Banach spaces (for then $X^{*}$ and $Y^{*}$ are Fréchet spaces and the open mapping theorem can still be applied).

We shall now construct proper identifications $(X, Y)$ where $Y$ is the Banach space $l^{q}, 1<q<\infty$, while $X$ is an inductive limit of Banach spaces. We shall denote by $l^{p}, 1 \leqslant p<\infty$, the space of sequences of real or complex numbers $\mathbf{a}=\left(a_{n}\right)$ for which the quantity

$$
\|\mathbf{a}\|_{p}=\left[\sum_{n=1}^{\infty}\left|a_{n}\right|^{p}\right]^{1 / p}
$$

is finite. If $p=\infty, l^{\infty}$ denotes the space of bounded sequences, with norm

$$
\|\mathbf{a}\|_{\infty}=\sup \left\{\left|a_{n}\right|: n \in \mathbf{N}\right\} \text {. }
$$

The spaces $l^{p}(1 \leqslant p<\infty)$, with the norm (3.1), are Banach spaces whose duals can be identified with $l^{p^{\prime}}$, where $1 / p+1 / p^{\prime}=1$. The duality between $l^{p}$ and $l^{p^{\prime}}$ is given by

$$
\langle\mathbf{a}, \mathbf{b}\rangle=\sum_{n=1}^{\infty} a_{n} b_{n}, \quad \mathbf{a} \in l^{p}, \mathbf{b} \in l^{p^{\prime}} .
$$

If $1 \leqslant p<q<\infty$, then $l^{p} \subset l^{q}$, the inclusion being proper, continuous and with dense image. For each set $E \subseteq \mathbf{N}$ with null density we denote by $X(p, q, E)$ the space of sequences a such that the quantity

$$
\|a\|_{p, q, E}=\left[\sum_{n \in E^{\prime}}\left|a_{n}\right|^{p}\right]^{1 / p}+\left[\sum_{n \in E}\left|a_{n}\right|^{q}\right]^{1 / q}
$$

is finite, where $E^{\prime}=\mathbf{N} \backslash E$. With the norm (3.4) the space $X(p, q, E)$ becomes a Banach space and as it is clear that

$$
l^{p} \subset X(p, q, E) \subset l^{q} .
$$


If $F$ is another set of null density with $E \subseteq F$, then

$$
X(p, q, E) \subseteq X(p, q, F)
$$

and the inclusion is continuous. When $E$ varies over the ring of sets of null density, an inductive system of Banach spaces is obtained. We shall denote the limit of such an inductive system as $X(p, q)$ :

$$
X(p, q)=\lim _{\vec{E}} X(p, q, E) .
$$

From the construction of $X(p, q)$, it follows that

$$
l^{p} \subseteq X(p, q) \subseteq l^{q} .
$$

A sequence $\mathbf{a} \in l^{q}$ belongs to $X(p, q)$ if and only if there is a set of null density $E$ such that

$$
\sum_{n \in E^{\prime}}\left|a_{n}\right|^{p}<\infty .
$$

Using this characterization of the elements of $X(p, q)$ we can show that $X(p, q)$ is a proper subspace of $l^{q}$. Take, for instance, $a_{n}=n^{-1 / p}$; clearly a $\in l^{q}$, but as we shall see a does not belong to $X(p, q)$. In fact, if $F \subseteq \mathbf{N}$ is any set for which the lower density $\underline{d}(F)>0$, then

$$
\sum_{n \in F}\left|a_{n}\right|^{p}=\infty .
$$

To see it, we order the elements of $F$ as a sequence $F=\left\{n_{1}, n_{2}, n_{3}, \ldots\right\}$, where $n_{1}<n_{2}<n_{3}<\cdots$. Since $\underline{d}(F)>0$, there are numbers $\varepsilon>0$ and $k_{0} \in \mathbf{N}$ such that $k / n_{k}>\varepsilon$ if $k \geqslant k_{0}$. Thus, $a_{n_{k}}^{p}=1 / n_{k}>\varepsilon / k$, and so

$$
\sum_{n \in F}\left|a_{n}\right|^{p} \geqslant \sum_{k=k_{0}}^{\infty} \frac{1}{n_{k}} \geqslant \varepsilon \sum_{k=k_{0}} \frac{1}{k}=\infty \text {. }
$$

THEOREM 2. The pair $\left(X(p, q), l^{q}\right)$ is a proper identification.

Proof. The only thing that remains to be proved is that the dual spaces of $X(p, q)$ and $l^{q}$ coincide.

But,

$$
l^{q^{\prime}}=\left(l^{q}\right)^{*} \subseteq X^{*}(p, q) \subseteq l^{p^{\prime}},
$$

where $1 / q+1 / q^{\prime}=1$ and $1 / p+1 / p^{\prime}=1$. Also, since $X(p, q)$ is the inductive limit of the spaces $X(p, q, E)$, it follows that a sequence $\mathbf{b} \in l^{p^{\prime}}$ belongs to $X^{*}(p, q)$ if and only if for every set of null density $E$ the series $\sum_{n \in E}\left|b_{n}\right|^{q^{\prime}}$ converges. We can now appeal to Theorem 1 to conclude that $\sum_{n=1}^{\infty}\left|b_{n}\right|^{q^{\prime}}$ converges also. Therefore, $\mathbf{b} \in l^{q^{\prime}}$.

\section{REFERENCES}

1. S. K. Berberian, Lectures in functional analysis and operator theory, Springer-Verlag, New York, 1974.

2. J. L. Kelley and I. Namioka, Linear topological spaces, Van Nostrand, Princeton, N.J., 1963.

Escuela de Matemática, Universidad de Costa Rica, San Jose, Costa Rica

Department of Mathematics, Pennsylvania State University, University Park, Pennsylvania 16802 